\title{
Crossover from intravalley to intervalley vortex motion in type-II superconductors with a periodic pinning array
}

\author{
S. Raedts, A. V. Silhanek, and V. V. Moshchalkov \\ Nanoscale Superconductivity and Magnetism Group, Laboratory for Solid State Physics and Magnetism, K. U. Leuven, \\ Celestijnenlaan 200 D, B-3001 Leuven, Belgium \\ J. Moonens and L. H. A. Leunissen \\ IMEC vzw, Kapeldreef 75, B-3001 Leuven, Belgium \\ (Received 24 December 2004; revised manuscript received 1 February 2006; published 15 May 2006)
}

\begin{abstract}
We have determined the transition from intravalley vortex motion (Campbell regime) to intervalley motion (critical state regime) in $\mathrm{Pb}$ thin films with and without a square array of holes (antidots) by means of ac susceptibility $\chi(T, H)$ measurements. The Campbell regime is characterized by a maximum dissipation $\chi_{\max }^{\prime \prime}$ dependent on the ac excitation $h$ but nearly temperature independent. In contrast, in the critical state regime, the height of the dissipation peak remains constant, whereas its position shifts to lower temperatures with increasing $h$. We introduce an alternative way for determining the temperature dependence of the ac onset of the Bean critical state by analyzing the critical current density $J(T)$ extracted from the $\chi^{\prime}(T)$ data at several $h$. We demonstrate that the presence of a periodic pinning array strongly affects the extension of the crossover area in the $h-T$ diagram between these regimes. We show that this effect can be ascribed to the lower dispersion of the pinning energy together with the higher topological order for the antidot sample.
\end{abstract}

DOI: $10.1103 /$ PhysRevB.73.174514

PACS number(s): 74.78.Na, 74.25.Sv, 74.78.Db, 74.25.Qt

\section{INTRODUCTION}

Superconductivity is commonly introduced in textbooks in a static way, as a lossless diamagnetic response. However, most practical applications involve ac currents, either at low frequencies in power lines or at high frequencies in microwave and computer applications. ${ }^{1}$ From the point of view of experimental techniques, the spectroscopic analysis of the sample's response to an external ac excitation provides rich information about the dynamics of the system. For example, one of the most popular means of investigating vortex dynamics in the mixed state of type-II superconductors consists of measuring the reaction of the vortex lattice to an external periodic field. ${ }^{2-4}$ The resulting deformation of the vortex lattice at the surface propagates into the interior, pushed forward by the elasticity of the vortex lattice and slowed down by pinning and viscous drag.

This ac-susceptibility technique allows one to access different vortex dynamic regimes by simply changing either the sensing parameters such as the amplitude $h$ and frequency $f$ of the ac perturbation or the thermodynamic variables, such as dc field $H$ and temperature $T .^{2,5-9}$ At small-enough values of the external excitation $h$, vortices oscillate inside the pinning potentials leaving intact the initial vortex configuration. This gives rise to an $h$-independent screening together with a very low dissipation. ${ }^{10,11}$ In this so-called Campbell regime, it is possible to determine the average curvature of the pinning potential $\alpha_{L}$ assuming a parabolic potential well. ${ }^{6,7}$ For higher amplitudes, the average distance traveled by vortices as they follow the ac drive approaches the pinning range and a nonlinear response is observed. Further increasing the acamplitude vortices overcomes the pinning potential well and a critical state, characterized by a higher dissipation and a penetration depth proportional to the ac field amplitude, develops. In this regime the initial vortex configuration is strongly disturbed after the ac measurement. ${ }^{7,12}$ In a recent work, we have shown that it is precisely this invasive character of the measurements in the critical state (CS) which allows one to determine the critical current density $J .^{8}$

In this work, using the ac-susceptibility technique we identify the intravalley and intervalley motion of vortices by several different approaches, all of them showing good degree of consistency. These measurements demonstrate that the extension of the crossover between the Campbell regime, at low ac amplitudes, and the critical state regime, at high amplitudes, is substantially altered due to the presence of a periodic pinning array.

\section{EXPERIMENTAL DETAILS}

The experiments were conducted on a $\mathrm{Pb}$ thin film with a square antidot array of period $d=1.5 \mu \mathrm{m}$, which corresponds to a first matching field $H_{1}=\phi_{0} / d^{2}=9.2 \mathrm{Oe}$. The antidots have a square shape with a lateral size $b=0.8 \mu \mathrm{m}$. The thickness $t$, sample dimensions, and critical temperature $T_{c}$ of the used sample are $t=13.5 \mathrm{~nm}, 1.9 \mathrm{~mm} \times 2 \mathrm{~mm}$, and $T_{c}$ $=7.10 \mathrm{~K}$, respectively. A plain $\mathrm{Pb}$ film was coevaporated on which $M(T, H)$ magnetization measurements were performed in order to determine the superconducting-normal phase boundary. From this $T_{c}(H)$ boundary we estimated a superconducting coherence length $\xi(0) \sim 33 \pm 1 \mathrm{~nm}$. The used nanostructured superconducting $\mathrm{Pb}$ film was evaporated in a MBE Varian VT-118 system on top of a $\mathrm{Si} / \mathrm{SiO}_{2}$ substrate covered by a double polymethyl metacrylatelmethyl metacrylate resist layer in which a square lattice of square dots was defined by electron-beam lithography at Imec vzw. The e-beam system at IMEC is a VB6-HR system from Leica Microsystems Lithography equipped with a thermal field emission electron source running at $20-100 \mathrm{kV}$ provid- 


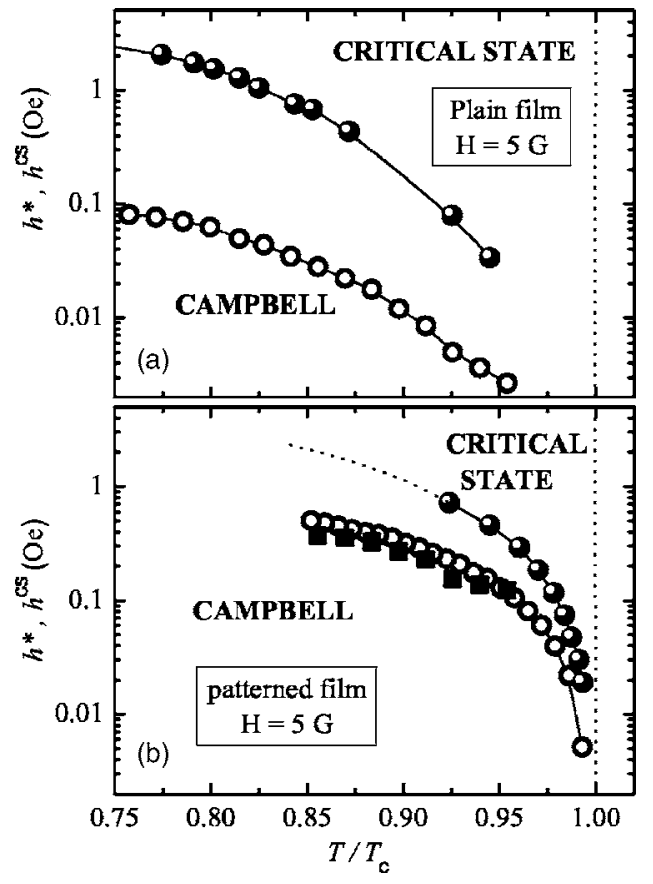

FIG. 1. Dynamic $h-T$ phase diagram for (a) a plain $\mathrm{Pb}$ film and (b) an antidot sample at $H=5$ Oe. Open circles: onset $h^{*}(T)$ of the nonlinear response. Filled circles: onset $h^{\mathrm{cs}}(T)$ of the critical state regime. The filled square symbols in the lower panel show the $h^{*}(T)$ boundary for the plain film multiplied by a factor $J($ antidots $) / J$ (plain film).

ing a high brightness and small source size. The system has a precision stage which can handle substrate sizes up to $200 \mathrm{~mm}$ wafers and stage positioning is monitored by a laser interferometer with $1.3 \mathrm{~nm}$ precision. After the evaporation, the resist is removed by a liftoff procedure leaving a $\mathrm{Pb}$ film with a square lattice of square holes (antidots). More details about the preparation procedure can be found in Ref. 13 .

The ac-susceptibility measurements $\chi=\chi^{\prime}+i \chi^{\prime \prime}$ were carried out in a commercial Quantum Design PPMS-device with the ac field $h$ parallel to the dc field $H$ and both applied perpendicular to the sample surface. The ac drive amplitude $h$ can be swept between $2 \mathrm{mOe}$ and $15 \mathrm{Oe}$, and the frequency $f$ from $10 \mathrm{~Hz}$ to $10 \mathrm{kHz}$. We report results obtained at a single frequency $f=3837 \mathrm{~Hz}$. In all cases, the data were normalized by the same factor corresponding to a total step $\Delta \chi^{\prime}=1$ with $H=0$.

\section{BOUNDARY OF THE CAMPBELL REGIME}

In order to identify the different dynamic regimes, we measure $\chi=\chi^{\prime}+i \chi^{\prime \prime}$ as a function of the ac drive amplitude $h$ for several temperatures $T$ at fixed dc field $H$. No differences between field-cooling and zero field-cooling measurements were observed. At low amplitudes of the ac drive, small vortex oscillations induce a linear response (Campbell regime) characterized by an $h$-independent screening $\chi^{\prime}$ and a very small dissipation $\chi^{\prime \prime}{ }^{5,6}$ As the ac drive is increased, vortices may overcome the pinning well and the system enters into a more dissipative regime with an $h$-dependent screening. ${ }^{7}$ By using the criterion $\chi_{\text {onset }}^{\prime \prime}=0.01$, the $\chi(h)$ curves allow one to determine the onset $h^{*}(T)$ of nonlinearity. ${ }^{8}$ The resulting boundaries $h^{*}(T)$ for the plain $\mathrm{Pb}$ film and for the antidot sample at $H=5$ Oe are shown as open symbols in Figs. 1(a) and 1(b), respectively.

Since the limits of the Campbell regime are mainly given by the strength of the pinning centers, it is expected that the extension of this dynamic phase decreases as temperature increases, in agreement with the observed behavior. It is also worth noticing that the extension of the Campbell regime for the patterned sample is clearly larger than for the reference film. Moreover, this result is quantitatively consistent with the predicted ${ }^{14}$ proportionality between $h^{*}$ and $J$. Indeed, the $h^{*}(T)$ boundary for the plain film multiplied by a factor $J$ (antidots) $/ J$ (plain film), represented by filled squares in Fig. 1(b), shows good agreement with the $h^{*}(T)$ for the patterned sample. This result is somehow surprising since the nature of the pinning centers, and therefore their shape, is different for the plain and the patterned film.

\section{CROSSOVER BETWEEN CAMPBELL AND CRITICAL STATE REGIME}

As we mentioned above, for $h>h^{*}$, vortices no longer oscillate locally around the pinning sites but perform displacements larger than the pinning range thus producing a nonlinear response. Further increasing $h$ beyond a threshold $h^{\mathrm{cs}}$, the system eventually enters in a regime such that the ac response can be described within a Bean critical state model with a uniform current density $J$ close to the critical value. Whereas in the Campbell regime, the characteristic ac penetration depth $\lambda_{\mathrm{ac}}$ is independent of $h$, in the critical state regime $\lambda_{\text {ac }}$ increases linearly with $h$ as ${ }^{15}$

$$
\lambda_{\mathrm{ac}}=\Lambda=\frac{c}{4 \pi} \frac{h}{J(T)} .
$$

For a given geometry, the ac response $\chi$ is solely determined by the penetration depth $\Lambda$ of the ac excitation. As a consequence, it is possible to find, within the critical state regime, a set of data at different $T$ and $h$ such that produce the same $\Lambda$ and consequently the same $\chi \cdot{ }^{8}$ According to Eq. (1), $\chi(h)$ measurements at constant $T$ within a welldeveloped critical state regime should collapse on a single curve when the horizontal axis $h$ is scaled by a suitable factor $J(T)^{-1}$. The resulting curve is $\chi^{\prime}(\Lambda)$ with $\Lambda$ the Bean penetration depth. At low screening values the curves show a good overlap, whereas a breakdown of the scaling is observed at higher screening values where the Campbell regime is approached. From the penetration depth $\Lambda_{\text {cross }}$ corresponding to this crossover and the obtained scaling parameters $J(T)$, we can estimate the onset of the critical state regime $h^{\mathrm{cs}}=\frac{4 \pi}{c} \Lambda_{\text {cross }} J(T)$. This boundary is represented by filled circles in Fig. 1 for both the plain and the patterned films. This figure shows that the transition between the Campbell and the critical state regime is not a sharp crossover, but instead, a broad intermediate nonlinear regime lies in between.

Let us now analyze the different mechanisms that may give rise to this crossover regime. First, due to the inhomo- 


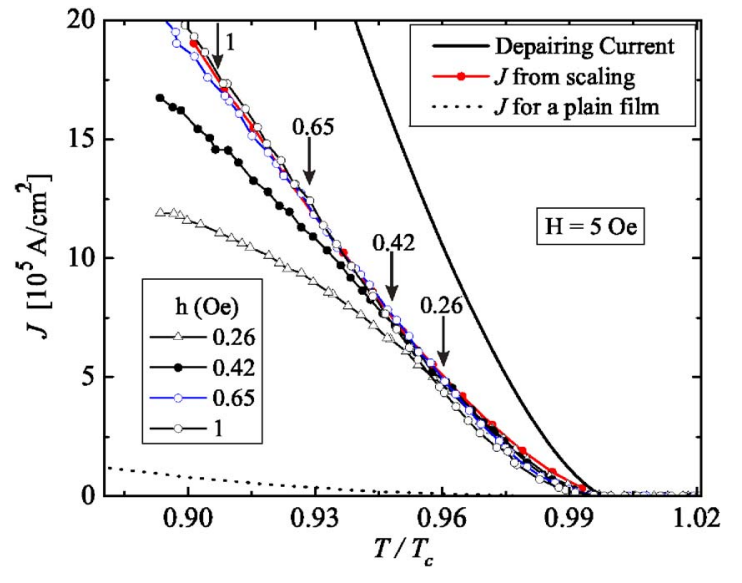

FIG. 2. (Color online) Temperature dependence of the critical current for the antidot sample extracted from the $\chi^{\prime}(T)$ curves shown in Fig. 4 at four different amplitudes $h$. The onset of the critical state regime, as given in Fig. 1(b), is indicated with a black arrow for each $h$ value. The red curve shows the critical current derived from the scaling procedure described in Ref. 8. The solid line depicts the depairing current density according to Ref. 19 and the dashed line indicates the $J(T)$ for the plain film.

geneous current distribution, nonlinearities first appear at the border of the sample and then, as $h$ increases, the boundary separating inter- and intravalley vortex motion shifts toward the center of the sample. This coexistence between Campbell and nonlinear regime gives rise to a nonlinear response. If this were the dominant scenario, since the field needed to achieve full penetration in the critical state is $\Delta h \sim J t$, whereas the thickness $t$ is the same for both samples, one expects that the width of the crossover regime increases by a factor $J$ (antidots) $/ J$ (plain film). However, a direct measurement of $J$ for both samples (see Fig. 2) gives $J($ antidots $) / J($ plain film $) \sim 20$ at $T / T_{c}=0.9$, whereas $\Delta h$ increases only by a factor of 2.5 at this temperature. Thus, we can rule out this effect as the main factor determining the width of the crossover regime.

An alternative possibility is that nonlinearities appear as a consequence of the anharmonicity of the pinning potential. Since the nature of the pinning in the antidot sample is completely different from that in the plain film, in principle, the onset where anharmonicities become relevant should also differ. Under this circumstance it is expected that the proportionality between $h^{*}(T)$ and $J$, deduced within an harmonic approximation, breaks down. In other words, the ratio $h^{*}(T) / J(T)$ is different for the plain film and the patterned sample. However, as we showed above this relationship is closely satisfied in the whole temperature range studied.

Still a further expansion of the intermediate regime may also occur as a consequence of the disorder in the distribution and energy of pinning centers. Since the disorder mainly affects the collective pinning properties of the system, it should have a strong influence on the onset of the critical state regime but little effect on the $h^{*}(T)$ boundary. Thus, this effect should manifest itself as a different evolution of $h^{*}$ and $h^{\text {cs }}$ as the system goes from weak pinning (plain film) to strong pinning (antidot sample). It is also expected that the

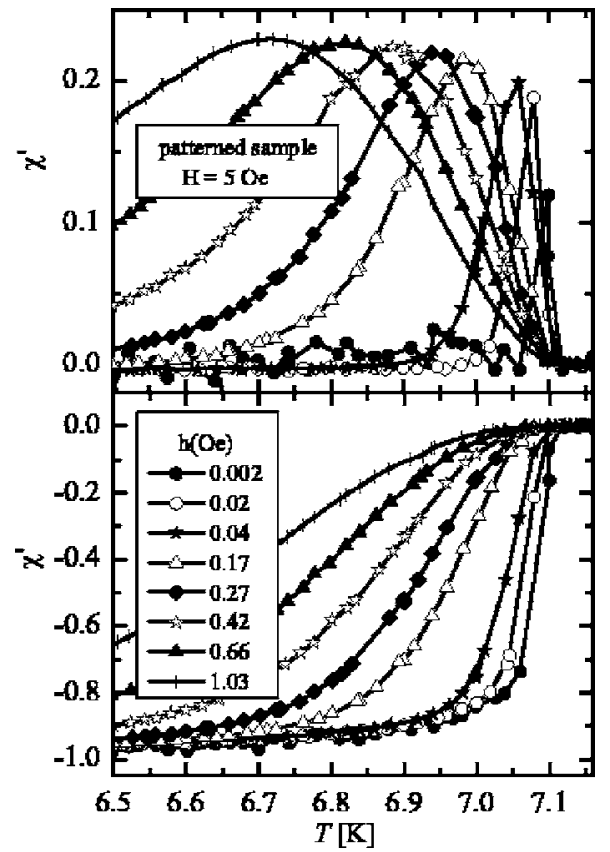

FIG. 3. Screening $\chi^{\prime}$ and dissipation $\chi^{\prime \prime}$ as function of temperature at different ac fields $h$ for the antidot sample.

increment in the average pinning intensity shifts up both boundaries in roughly the same amount, however we found that $h^{\text {cs }}$ increases only by a factor of 2 whereas $h^{*}$ rises by a factor of 20. These findings indicate that in patterned samples, where a regular array of pinning centers dramatically reduces the disorder, the $h^{\text {cs }}$ boundary approaches the $h^{*}$ border.

An alternative way to identify the boundary $h^{*}$ between the Campbell and nonlinear regime and to estimate the onset $h^{\mathrm{cs}}$ of the critical state regime consists of tracking the $h$ and $T$ evolution of the $\chi^{\prime \prime}$ peak. Indeed, whereas in the flux flow regime $\chi_{\max }^{\prime \prime} \sim 0.44$, for a critical state regime $\chi_{\max }^{\prime \prime}$ $\sim 0.24 .{ }^{15-17}$ On the other hand, $\chi_{\max }^{\prime \prime}$ may also adopt values between these two in the dissipative creep regime or $\chi_{\max }^{\prime \prime}$ $\rightarrow 0$ as the system approaches the Campbell regime.

Additionally, in the critical state regime the height of the dissipation peak remains weakly $h$ dependent, whereas its position shifts down in $H$ and $T$ as $h$ increases. In contrast, in the Campbell regime it is expected that $\chi_{\max }^{\prime \prime}$ increases with increasing $h$ as the system approaches the boundary of the nonlinear regime, whereas the peak position remains unchanged. This behavior is clearly seen in Fig. 3 where we show the temperature dependence of the screening $\chi^{\prime}$ and dissipation $\chi^{\prime \prime}$ for a series of different ac fields $h$ for the antidot sample. Here, it can be observed that the height of the peak $\chi_{\max }^{\prime \prime}$ grows at low amplitudes and saturates to a constant value for higher amplitudes. A detailed analysis of the peak evolution is shown in Figs. 4(a) and 4(b) where the height $\chi_{\max }^{\prime \prime}$ and the position $T_{\max }$ of the maximum dissipation are plotted as a function of $h$. In Fig. 4(a), we observe a crossover from a strongly $h$-dependent $\chi_{\max }^{\prime \prime}$ in the Campbell regime to an almost independent $\chi_{\max }^{\prime \prime} \sim 0.24$ corresponding to the critical state regime. Similar results were reported by Pasquini et al..$^{5}$ and Herzog et al. ${ }^{16}$ in high- $T_{c}$ superconductors. 


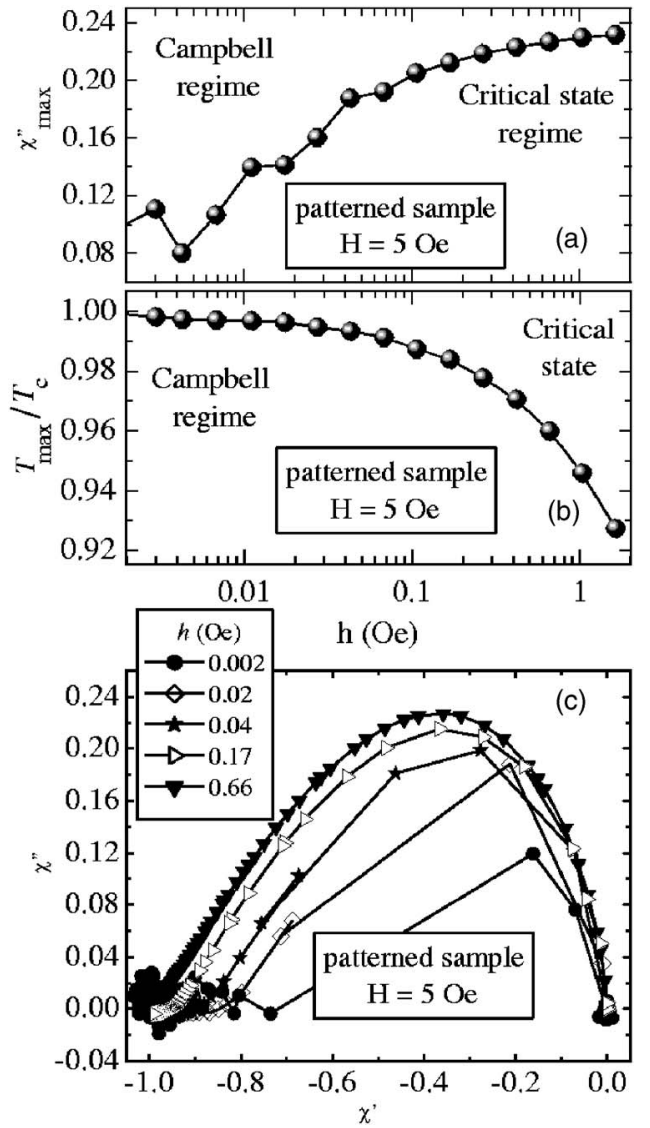

FIG. 4. (a) Height of the peak in $\chi^{\prime \prime}(T)$ as a function of the ac field $h$. (b) Temperature $T_{\max }$ at which the peak in $\chi^{\prime \prime}(T)$ occurs. (c) Cole-Cole plot of the data shown in Fig. 3.

As we pointed out previously, the position of the peak in $\chi^{\prime \prime}$ shifts toward lower temperatures as $h$ increases as it can be seen in Fig. 3. This behavior is shown in Fig. 4(b), where the peak position $T_{\max }$ tends to saturate at a constant value at low amplitudes whereas it evolves to an $h$-dependent $T_{\max }$ as $h$ increases. This is in agreement with a critical state model according to which the maximum in $\chi^{\prime \prime}$ is attained at a temperature $T_{\max }$ such that $\Lambda \sim t$, with $t$ the sample thickness. Using Eq. (1), it follows that to keep $\Lambda \sim t$, an increment of the ac drive $h$ results in a shift of $T_{\max }$ to lower values such that $J(T)$ increases. All these effects become evident in Fig. 4(c), where a Cole-Cole plot of the data presented in Fig. 3 is shown. In Fig. 3, a clear evolution from an almost nondissipative regime to a highly dissipative regime in the critical state can be observed.

\section{TEMPERATURE DEPENDENCE OF THE CRITICAL CURRENT}

Let us now determine to which extent the Bean critical state model can be applied to describe the susceptibility data at high ac amplitudes. According to the Bean critical state model, if both $H$ and $h$ are applied perpendicular to the plane of a strip, the screening is given by ${ }^{16}$

$$
\chi^{\prime}=-\frac{\tan h(s)}{s},
$$

where $s=h / H_{d} \propto \Lambda / t \propto J_{c}^{-1}$ and $H_{d}$ the characteristic field for this particular sample geometry. ${ }^{18}$ Thus, using this equation, we can estimate from the $\chi^{\prime}(T)$ data, shown in Fig. 3, the temperature dependence of the critical current density $J(T)$. The result of this analysis is presented in Fig. 2 for four different ac fields.

Within this model, $J$ is independent of the used ac field $h$, and therefore, the obtained critical current for different $h$ should coincide in the temperature range where the Bean approximation holds. In Fig. 2, it can be clearly seen that the obtained $J(T)$ curves overlap for temperatures close to $T_{c}$ and depart from this universal behavior as temperature decreases (see black arrows). In other words, the Bean critical state model fails for temperatures and ac fields such that $h<h^{\mathrm{cs}}(T)$, with $h^{\mathrm{cs}}(T)$ the onset of the critical state regime, as shown in Fig. 1. Naturally, the higher the ac field $h$, the broader the temperature range in which the Bean critical state model is valid. It is worth noticing that the black arrows in Fig. 2, indicating the boundary $h^{\mathrm{cs}}(T)$, coincide well with the independently determined departure of the universal behavior of $J(T)$.

Figure 2 also shows the critical current density $J(T)$ (red curve) obtained from the scaling procedure described in Ref. 8 . The remarkable coincidence of the $J(T)$ data in the temperature range where the Bean critical state model is valid confirms the precise character of the scaling procedure. Finally, it is worth mentioning that the $J(T)$ curves for $h$ $\Im 0.65$ Oe coincide over a substantial temperature range in which most of our ac susceptibility measurements were performed. Consistently, as can be seen in Figs. 4(a) and 4(b), for $h>0.65$ Oe the height of the peak in the $\chi^{\prime \prime}(T)$ seems to saturate whereas the temperature at which the peak occurs is clearly $h$ dependent.

\section{CONCLUSION}

A direct comparison of the boundary between the Campbell and critical state regime for both a plain $\mathrm{Pb}$ film and an antidot film, shows that the width of the crossover area between these regimes in the $h-T$ diagram is strongly affected by the presence of a regular array of pinning centers. We show that this intermediate regime is influenced by the energy dispersion and the topological order of the pinning landscape. We were able to identify the different dynamic regimes by tracking the position and height of the maximum in the dissipation $\chi_{\max }^{\prime \prime}$ for different ac drives. Finally, we extracted the critical current density $J(T)$ from the $\chi^{\prime}(T)$ using a theoretical approximation for a strip geometry within the Bean critical state model and found a good agreement with the $J(T)$ independently determined from the scaling procedure described in Ref. 8. This indicates the validity of the Bean CS model for temperatures ranging from $0.9 T_{c}$ to $T_{c}$ depending on the applied ac field $h$. 


\section{ACKNOWLEDGMENTS}

The authors would like to thank M. J. Van Bael for helpful discussions. This work was supported by the Fund for Scientific Research-Flanders (FWO-Vlaanderen), the Belgian
Inter-University Attraction Poles (IUAP), the Research Fund K.U. Leuven GOA/2004/02 and by the European ESF VORTEX programs. S.R. acknowledge support from the Research Council of the K.U. Leuven and A.V.S. is grateful for the support from the FWO-Vlaanderen.
${ }^{1}$ http://www.superconductors.org/Uses.htm. See also C. A. Hamilton, F. L. Lloyd, R. L. Peterson, and J. R. Andrews, Appl. Phys. Lett. 35, 718 (1979).

${ }^{2}$ C. J. Van der Beek, V. B. Geshkenbein, and V. M. Vinokur, Phys. Rev. B 48, 3393 (1993).

${ }^{3}$ F. Gömöry, Supercond. Sci. Technol. 10, 523 (1997).

${ }^{4}$ E. H. Brandt, Physica C 195, 1 (1992).

${ }^{5}$ G. Pasquini, P. Levy, L. Civale, G. Nieva, and H. Lanza, Physica C 274, 165 (1997).

${ }^{6}$ A. V. Silhanek, S. Raedts, M. Lange, and V. V. Moshchalkov, Phys. Rev. B 67, 064502 (2003).

${ }^{7}$ G. Pasquini, L. Civale, H. Lanza, and G. Nieva, Phys. Rev. B 59, 9627 (1999); L. Civale, A. V. Silhanek, and G. Pasquini, Studies of High Temperature Superconductors, edited by Anant Narlikar, International Book Series Vol. 47 (Nova Science Publishers, New York, 2003).

${ }^{8}$ A. V. Silhanek, S. Raedts, M. J. Van Bael, and V. V. Moshchalkov, Eur. Phys. J. B 37, 19 (2004).

${ }^{9}$ A. D. Hernández and D. Domínguez, Phys. Rev. B 66, 144505
(2002).

${ }^{10}$ A. M. Campbell and J. E. Evetts, Adv. Phys. 21, 199 (1972).

${ }^{11}$ A. M. Campbell and J. E. Evetts, Adv. Phys. 50, 1249 (2001).

${ }^{12}$ S. O. Valenzuela and V. Bekeris, Phys. Rev. Lett. 84, 4200 (2000).

${ }^{13}$ S. Raedts, A. V. Silhanek, M. J. Van Bael, and V. V. Moshchalkov, Phys. Rev. B 70, 024509 (2004).

${ }^{14}$ G. Pasquini, L. Civale, H. Lanza, and G. Nieva, Phys. Rev. B 65 , 214517 (2002).

${ }^{15}$ J. R. Clem and A. Sanchez, Phys. Rev. B 50, 9355 (1994).

${ }^{16}$ Th. Herzog, H. A. Radovan, P. Ziemann, and E. H. Brandt, Phys. Rev. B 56, 2871 (1997).

${ }^{17}$ D. V. Shantsev, Y. M. Galperin, and T. H. Johansen, Phys. Rev. B 61, 9699 (1993).

${ }^{18}$ Using the equation corresponding to a disk geometry leads to an absolute value of $J_{c}$ about $15 \%$ smaller than the one obtained for a strip geometry.

${ }^{19}$ M. N. Kunchur, D. K. Christen, and J. M. Phillips, Phys. Rev. Lett. 70, 998 (1993). 